\title{
The epidemiological characteristics of pediatric Streptococcus pneumoniae isolated from inpatients and outpatients at Beijing Children's Hospital
}

Shuang Lyu ( $\sim$ Ivshuanghe2007@126.com )

\section{Research article}

Keywords: Streptococcus pneumoniae, serotype distribution, antibiotic resistance, multilocus sequence typing

Posted Date: June 19th, 2020

DOI: https://doi.org/10.21203/rs.3.rs-35966/v1

License: (c) (i) This work is licensed under a Creative Commons Attribution 4.0 International License. Read Full License 


\section{Abstract}

Background: The epidemiological data of Streptococcus pneumoniae isolates are important for the practice of treatment and prevention. This research aiming to explore the epidemiological characteristics of pediatric $S$. pneumoniae isolated from outpatients and inpatients.

Methods: S. pneumoniae were isolated from unsterile samples of inpatients and outpatients younger than five years old between March 2013 and February 2014. The serotypes were determined by diagnostic pneumococcal antisera, and resistance against 13 antibiotics was tested by either the E-test or the disc diffusion method. The sequence types (STs) were analyzed with multilocus sequence typing (MLST).

Results: The five dominant serotypes obtained from inpatients were $19 \mathrm{~F}(32.9 \%), 19 \mathrm{~A}(20.7 \%), 23 \mathrm{~F}(10.7 \%)$, $6 \mathrm{~A}(10.0 \%)$, and $14(8.6 \%)$, while those in the outpatients were $19 \mathrm{~F}(13.6 \%), 23 \mathrm{~F}(12.9 \%), 6 \mathrm{~A}(10.0 \%), 6 \mathrm{~B}$ $(10.0 \%)$, and $19 \mathrm{~A}(7.9 \%)$. The coverage rates of the $7-, 10$ - and 13 -valent pneumococcal vaccine formulations were high. The non-susceptibility to penicillin, cefuroxime, imipenem, erythromycin, and trimethoprim-sulfamethoxazole among the inpatient isolates were $7.1 \%, 92.8 \%, 65.7 \%, 100 \%$, and $85.0 \%$, respectively, while those among the outpatient isolates were $0.7 \%, 50.0 \%, 38.6 \%, 96.4 \%$, and $65.7 \%$, respectively. There were 45 and 81 STs detected from the pneumococci from inpatients and outpatients, respectively. CC271 was more prevalent in inpatients.

Conclusions: The vaccine related serotypes were still prevalent either in inpatient department or in outpatient department, the prevalent serotypes and MLSTs with serious antibiotic resistance. More effective measures should be implemented to prevent S. pneumoniae diseases.

\section{Background}

Streptococcus pneumoniae (S. pneumoniae) is a major pathogen of infectious diseases worldwide. The epidemiological data of invasive $S$. pneumoniae isolates are important for the practice of treatment and prevention. However, it is hard to collect an adequate number of isolates in countries where the rate of positive bacteria cultures from invasive samples are very low. We collected 171 invasive pneumococcal isolates from 11 hospitals between 2006 and 2008 [1]. On average, only 5.2 isolates are collected in each hospital every year, which limits the representation of this pathogen in these regions. The Centers for Disease Control and Prevention (CDC) and the World Health Organization (WHO) recommended that nasopharyngeal isolates could be used for the surveillance of pneumococcal epidemiology since they could be obtained in higher number [2,3]. However, in Chinese hospitals, the nasopharyngeal swab collection is not a regular work and is only used for some research projects. Such collection will be stopped when the project is finished. In China and other developing countries, etiologic examination is usually performed to inpatients. Therefore, the bacterial culturing of sputum (i.e., laryngohypopharynx aspirates) and bronchoalveolar lavage can be continuously obtained in the daily clinical work at the inpatient department, which is an important facilitating condition for continuous surveillance. 
In our two previous studies, higher drug resistance and an increased 7-valent pneumococcal conjugate vaccine (PCV7) related serotype coverage rate were found in isolates from the hypopharyngeal aspirates of inpatients with pneumonia than those of nasopharyngeal isolates from nasopharyngeal swabs of outpatients with an upper respiratory infection $[4,5]$. However, the two studies were not completed during the same period. The exact epidemiological characteristics of $S$. pneumoniae isolated from pediatric inpatients and outpatients in the same peroid could not be found in previous reports.

In the present study, clinical non-invasive S. pneumoniae isolates from inpatients and nasopharyngeal carriage isolates from outpatients visiting Beijing Children's Hospital were collected between March 2013 and February 2014. The serotype distribution, PCV coverage rate, antimicrobial resistance, and MLST were analyzed for these two groups.

\section{Methods}

The current study cohort was composed of the children younger than 5 years old in Beijing Children's Hospital between March 2013 and February 2014. Inpatients were children with pneumonia who were admitted into the Infectious Diseases Department, the Respiratory Diseases Department, and the Intensive Care Unit. Outpatients were children who visited the Outpatient Department with a respiratory infection.

All the inpatient isolates were cultured in a clinical laboratory following a procedure similar to succedent annotation [6]. The isolates were transported to the Microbial Laboratory for further tests. For the outpatients, nasopharyngeal swabs were collected and immediately transported to the Microbial Laboratory. The samples were inoculated within 3-4h onto tryptone soy agar plates with $5 \%$ sheep blood containing $5 \mathrm{~g} / \mathrm{ml}$ gentamicin. The plates were incubated at $35^{\circ} \mathrm{C}$ under a $5 \% \mathrm{CO}_{2}$ atmosphere and examined after 18-24 h. Using the random number table method, arbitrary outpatient isolates were selected monthly to correspond to the same number of inpatient isolates.

As previously described in detail [6], all the isolates were identified based on the typical colony morphology, Gram staining, an optochin sensitivity test (Oxoid Company, Britain), and an Omni serum assay (Statens Serum Institut, Copenhagen, Denmark). All isolates were stored at $-80^{\circ} \mathrm{C}$ in freezing tubes for further study. Only one isolate from each participant was included in the present study.

As previously described in detail [6], the serogroups were tested using the Quellung reaction with Pneumotest kits, and the serotypes were tested with factor antisera (Statens Serum Institute, Copenhagen, Denmark). The interpretation of the serotyping depended on the capsular swelling under phase-contrast microscopy with an oil immersion lens (magnification, 100x) as described in the literature [7]. The serotype coverage rates of the PCV7, PCV10, and PCV13 were estimated by calculating the percentage of isolates that expressed the serotypes included in the vaccines.

For all isolates, the minimum inhibitory concentrations (MICs) were determined against penicillin, erythromycin, amoxicillin-clavulanic acid, cefaclor, cefuroxime, ceftriaxone, levofloxacin, linezolid, 
vancomycin and imipenem using E-test strips (BIOMERIEUX, France), and the antimicrobial susceptibilities to chloramphenicol, tetracycline and trimethoprim-sulfamethoxazole were determined using the Kirby-Bauer disk diffusion test (OXOID Company, Britain). The Clinical and Laboratory Standards Institute's (CLSI) 2015 criteria for MICs were applied to classify isolates as susceptible, intermediate, or resistant [8]. As previously described in detail [6], the S. pneumoniae American Type Culture Collection strain 49619 (ATCC 49619) was used as a quality control strain and was included in each set of tests to ensure the accuracy of the results. Multi-drug resistant $S$. pneumoniae (MDRSP) were defined as resistant to three or more classes of antibiotics tested in this study.

The housekeeping genes aroE, gdh, gki, recP, spi, xpt, and $d d l$ were amplified via polymerase chain reaction (PCR) [9]. The sequences of seven loci were each compared with those of all known alleles at the loci as well as with the STs in the database of the pneumococcal MLST website (https://pubmlst.org/spneumoniae). New allelic numbers or new ST numbers were assigned by the curator of the pneumococcal MLST website [10]. eBURST v3 software (https://eburst.mlst.net//) was used to investigate the relationships between the isolates and to assign a clonal complex (CC) based on the stringent group definition of six out of seven shared alleles. STs that shared six identical alleles among the seven MLST loci with another ST in the group were reclassified into one group as a CC.

\section{Statistical analysis}

The antimicrobial resistance data was collected and analyzed using WHONET 5.6 software as recommended by the WHO, and the serotype data was collected and analyzed by Excel 2017.

\section{Results}

During the study period, 140 S. pneumoniae isolates were collected from inpatients which were cultured from hypopharyngeal aspirates $(n=104)$, bronchoalveolar lavage $(n=33)$, nasopharyngeal swabs $(n=1)$ or ear discharge $(n=2)$. Of the 693 pneumococcal strains isolated from outpatients, 140 were randomly selected using random number table method.

\section{Serotype distribution and Vaccine coverage}

The serotype distribution and vaccine coverage between the inpatient and outpatient S. pneumoniae isolates are shown in Table 1. The serotype was included in the table when its constituent ratio was greater than $5 \%$. The serotype distribution was more concentrated among the inpatient isolates, in which only 15 serotypes were identified. The most common serotypes were 19F (32.9\%), 19A (20.7\%), 23F (10.7\%), 6A (10.0\%), 14 (8.6\%) and 15B (6.4\%), which accounted for 89.3\%. Meanwhile, 29 serotypes were identified among the outpatient isolates. The most frequent serotypes were 19F (13.6\%), 23F (12.9\%), 6A (10.0\%), 6B (10.0\%), 19A (7.9\%) and 34 (5.0\%), which accounted for 59.3\%. In addition, the serotype coverage rates of PCVs among the inpatient isolates were high. 
Table 1

Serotype distribution and vaccines coverage rates of inpatient and outpatient $S$. pneumoniae isolates [n (\%)]

\begin{tabular}{|c|c|c|}
\hline $\begin{array}{l}\text { Serotype / } \\
\text { coverage rate }\end{array}$ & $\begin{array}{l}\text { Inpatient isolates } \\
(n=140)\end{array}$ & $\begin{array}{l}\text { Outpatient isolates } \\
(n=140)\end{array}$ \\
\hline $19 \mathrm{~F}$ & $46(32.9 \%)$ & $19(13.6 \%)$ \\
\hline $23 \mathrm{~F}$ & 15 (10.7\%) & $18(12.9 \%)$ \\
\hline 14 & $12(8.6 \%)$ & $5(3.6 \%)$ \\
\hline $6 \mathrm{~B}$ & $4(2.9 \%)$ & $14(10.0 \%)$ \\
\hline $9 \mathrm{~V}$ & $1(0.7 \%)$ & 0 \\
\hline $18 \mathrm{C}$ & 0 & $1(0.7 \%)$ \\
\hline $7 F$ & $1(0.7 \%)$ & $1(0.7 \%)$ \\
\hline $19 \mathrm{~A}$ & $29(20.7 \%)$ & $11(7.9 \%)$ \\
\hline $6 \mathrm{~A}$ & $14(10.0 \%)$ & $14(10.0 \%)$ \\
\hline 3 & $1(0.7 \%)$ & $3(2.1 \%)$ \\
\hline 15B & $9(6.4 \%)$ & $5(3.6 \%)$ \\
\hline 34 & 0 & $7(5.0 \%)$ \\
\hline Others & $8(5.7 \%)^{a}$ & $42(30.0 \%)^{b}$ \\
\hline PCV7 & 78 (55.7\%) & 57 (40.7\%) \\
\hline PCV10 & 79 (56.4\%) & $58(41.4 \%)$ \\
\hline PCV13 & 123 (87.9\%) & $86(61.4 \%)$ \\
\hline
\end{tabular}

\section{Antimicrobial Susceptibility Testing}

The antimicrobial susceptibilities of the inpatient and outpatient isolates are shown in Table 2. The nonsusceptibility rate of penicillin in inpatients was high, and the penicillin $\mathrm{MIC}_{50}$ and $\mathrm{MIC}_{90}$ values were also higher. Among the inpatient isolates, the non-susceptibility rate of amoxicillin-clavulanic acid, imipenem, cefuroxime, cefaclor and trimethoprim-sulfamethoxazole were $7.1 \%, 65.7 \%, 92.8 \%, 93.6 \%$ and $85 \%$, respectively. The corresponding date were $0.7 \%, 38.6 \%, 50 \%, 53.5 \%$ and $65.7 \%$, respectively. All of the isolates showed high non-susceptibility to erythromycin and tetracycline. The resistant rate of chloramphenicol was low both in inpatient isolates and outpatient isolates. All pneumococcal isolates were susceptible to vancomycin, linezolid and levofloxacin. 
Table 2

Susceptibility and MICs to 13 antibiotics of inpatient and outpatient S. pneumoniae isolates

\begin{tabular}{|c|c|c|}
\hline Antimicrobiala $^{a}$ & $\begin{array}{l}\text { Inpatient isolates } \\
(n=140)\end{array}$ & $\begin{array}{l}\text { Outpatient isolates } \\
(n=140)\end{array}$ \\
\hline \multicolumn{3}{|l|}{ Penicillin } \\
\hline \multicolumn{3}{|c|}{ Parenteral non-meningitis } \\
\hline $\mathrm{R} \%$ & 2.1 & 0.7 \\
\hline $1 \%$ & 5 & 0 \\
\hline \multicolumn{3}{|l|}{ Oral non-meningitis } \\
\hline $\mathrm{R} \%$ & 40.0 & 20.7 \\
\hline $1 \%$ & 54.3 & 38.6 \\
\hline MIC range (mg/L) & $0.016-12$ & $0.004-6$ \\
\hline $\mathrm{MIC}_{50}(\mathrm{mg} / \mathrm{L})$ & 1 & 0.5 \\
\hline $\mathrm{MIC}_{90}(\mathrm{mg} / \mathrm{L})$ & 2 & 1.5 \\
\hline \multicolumn{3}{|c|}{ Amoxicillin-clavulanic acid } \\
\hline $\mathrm{R} \%$ & 0.7 & 0 \\
\hline $1 \%$ & 6.4 & 0.7 \\
\hline MIC range (mg/L) & $0.016-12$ & $0.016-3$ \\
\hline $\mathrm{MIC}_{50}(\mathrm{mg} / \mathrm{L})$ & 1 & 0.38 \\
\hline $\mathrm{MIC}_{90}(\mathrm{mg} / \mathrm{L})$ & 2 & 1.5 \\
\hline \multicolumn{3}{|l|}{ Cefuroxime } \\
\hline $\mathrm{R} \%$ & 87.1 & 43.6 \\
\hline $1 \%$ & 5.7 & 6.4 \\
\hline MIC range (mg/L) & $0.023-48$ & $0.016-12$ \\
\hline $\mathrm{MIC}_{50}(\mathrm{mg} / \mathrm{L})$ & 3 & 0.5 \\
\hline
\end{tabular}

Notes:

a. The breakpoint of penicillin was based on the parenteral non-meningitis (intermediate [ $4 \mathrm{mg} / \mathrm{L}]$, resistant $[\geq 8 \mathrm{mg} / \mathrm{L}]$ ), and oral non-meningitis (intermediate $[0.12-1 \mathrm{mg} / \mathrm{L}]$, resistant $[\geq 2 \mathrm{mg} / \mathrm{L}]$ ). The breakpoint of ceftriaxone was based on only parenteral non-meningitis(intermediate [2 $\mathrm{mg} / \mathrm{L}]$, resistant $[\geq 4 \mathrm{mg} / \mathrm{L}])$. 


\begin{tabular}{|c|c|c|}
\hline Antimicrobiala & $\begin{array}{l}\text { Inpatient isolates } \\
(n=140)\end{array}$ & $\begin{array}{l}\text { Outpatient isolates } \\
(n=140)\end{array}$ \\
\hline $\mathrm{MIC}_{90}(\mathrm{mg} / \mathrm{L})$ & 8 & 4 \\
\hline \multicolumn{3}{|l|}{ Cefaclor } \\
\hline $\mathrm{R} \%$ & 90.7 & 51.4 \\
\hline $1 \%$ & 2.9 & 2.1 \\
\hline $\mathrm{MIC}$ range $(\mathrm{mg} / \mathrm{L})$ & $0.19->256$ & $0.125->256$ \\
\hline $\mathrm{MIC}_{50}(\mathrm{mg} / \mathrm{L})$ & 24 & 3 \\
\hline $\mathrm{MIC}_{90}(\mathrm{mg} / \mathrm{L})$ & 64 & 96 \\
\hline \multicolumn{3}{|l|}{ Ceftriaxone } \\
\hline $\mathrm{R} \%$ & 1.4 & 0.7 \\
\hline $1 \%$ & 17.9 & 10.7 \\
\hline $\mathrm{MIC}$ range (mg/L) & $0.012-4$ & $0.006-3$ \\
\hline $\mathrm{MIC}_{50}(\mathrm{mg} / \mathrm{L})$ & 0.75 & 0.38 \\
\hline $\mathrm{MIC}_{90}(\mathrm{mg} / \mathrm{L})$ & 1.5 & 1.5 \\
\hline \multicolumn{3}{|l|}{ Imipenem } \\
\hline $\mathrm{R} \%$ & 0.7 & 0 \\
\hline $1 \%$ & 65 & 38.6 \\
\hline $\mathrm{MIC}$ range $(\mathrm{mg} / \mathrm{L})$ & $0.008-2$ & $0.004-0.38$ \\
\hline $\mathrm{MIC}_{50}(\mathrm{mg} / \mathrm{L})$ & 0.19 & 0.094 \\
\hline $\mathrm{MIC}_{90}(\mathrm{mg} / \mathrm{L})$ & 0.25 & 0.25 \\
\hline \multicolumn{3}{|l|}{ Vancomycin } \\
\hline MIC range $(\mathrm{mg} / \mathrm{L})$ & $0.19-0.5$ & $0.25-1$ \\
\hline $\mathrm{MIC}_{50}(\mathrm{mg} / \mathrm{L})$ & 0.5 & 0.5 \\
\hline
\end{tabular}

Notes:

a. The breakpoint of penicillin was based on the parenteral non-meningitis (intermediate $[4 \mathrm{mg} / \mathrm{L}]$, resistant $[\geq 8 \mathrm{mg} / \mathrm{L}]$ ), and oral non-meningitis (intermediate $[0.12-1 \mathrm{mg} / \mathrm{L}]$, resistant $[\geq 2 \mathrm{mg} / \mathrm{L}])$. The breakpoint of ceftriaxone was based on only parenteral non-meningitis(intermediate [2 $\mathrm{mg} / \mathrm{L}]$, resistant $[\geq 4 \mathrm{mg} / \mathrm{L}])$. 


\begin{tabular}{|c|c|c|}
\hline Antimicrobial ${ }^{\mathrm{a}}$ & $\begin{array}{l}\text { Inpatient isolates } \\
(n=140)\end{array}$ & $\begin{array}{l}\text { Outpatient isolates } \\
(n=140)\end{array}$ \\
\hline $\mathrm{MIC}_{90}(\mathrm{mg} / \mathrm{L})$ & 0.5 & 0.5 \\
\hline \multicolumn{3}{|l|}{ Linezolid } \\
\hline MIC range (mg/L) & $0.38-2$ & $0.25-2$ \\
\hline $\mathrm{MIC}_{50}(\mathrm{mg} / \mathrm{L})$ & 0.75 & 1 \\
\hline $\mathrm{MIC}_{90}(\mathrm{mg} / \mathrm{L})$ & 1 & 1.5 \\
\hline \multicolumn{3}{|l|}{ Levofloxacin } \\
\hline MIC range (mg/L) & $0.38-2$ & $0.38-2$ \\
\hline $\mathrm{MIC}_{50}(\mathrm{mg} / \mathrm{L})$ & 0.75 & 0.75 \\
\hline $\mathrm{MIC}_{90}(\mathrm{mg} / \mathrm{L})$ & 1 & 1 \\
\hline \multicolumn{3}{|l|}{ Erythromycin } \\
\hline $\mathrm{R} \%$ & 100 & 96.4 \\
\hline MIC range (mg/L) & $4->256$ & $0.094-256$ \\
\hline $\mathrm{MIC}_{50}(\mathrm{mg} / \mathrm{L})$ & $>256$ & $>256$ \\
\hline $\mathrm{MIC}_{90}(\mathrm{mg} / \mathrm{L})$ & $>256$ & $>256$ \\
\hline \multicolumn{3}{|l|}{ Tetracycline } \\
\hline $\mathrm{R} \%$ & 92.9 & 93.6 \\
\hline $1 \%$ & 4.2 & 1.4 \\
\hline \multicolumn{3}{|l|}{ Chloramphenicol } \\
\hline $\mathrm{R} \%$ & 4.3 & 10.7 \\
\hline \multicolumn{3}{|c|}{ Trimethoprim-sulfamethoxazole } \\
\hline $\mathrm{R} \%$ & 85 & 65.7 \\
\hline $1 \%$ & 2.1 & 11.4 \\
\hline \multicolumn{3}{|l|}{ Notes: } \\
\hline \multicolumn{3}{|c|}{$\begin{array}{l}\text { a. The breakpoint of penicillin was based on the parenteral non-meningitis (intermediate }[4 \mathrm{mg} / \mathrm{L}] \text {, } \\
\text { resistant }[\geq 8 \mathrm{mg} / \mathrm{L}]) \text {, and oral non-meningitis (intermediate }[0.12-1 \mathrm{mg} / \mathrm{L}] \text {, resistant }[\geq 2 \mathrm{mg} / \mathrm{L}]] \text {. The } \\
\text { breakpoint of ceftriaxone was based on only parenteral non-meningitis(intermediate }[2 \mathrm{mg} / \mathrm{L}] \text {, } \\
\text { resistant }[\geq 4 \mathrm{mg} / \mathrm{L}] \text { ). }\end{array}$} \\
\hline
\end{tabular}


The multi-drug resistant pattern of the pneumococcal isolates is shown in the Table 3 . Approximately $92.1 \%(129 / 140)$ of all inpatient isolates and $85.0 \%$ (119/140) of all outpatient isolates were MDRSP. The most prevalent antibiotic resistant pattern of macrolides/ $\beta$-lactams/tetracyclines/sulfonamides was observed both in inpatient and outpatient isolates.

Table 3

Multi-drug resistant pattern of inpatient and outpatient S. pneumoniae isolates [n (\%)]

\begin{tabular}{|c|c|c|c|}
\hline $\begin{array}{l}\text { Class of } \\
\text { antibiotic }\end{array}$ & Resistance pattern* & $\begin{array}{l}\text { Inpatient } \\
\text { isolates } \\
(n=140)\end{array}$ & $\begin{array}{l}\text { Outpatients } \\
\text { isolates } \\
(n=140)\end{array}$ \\
\hline 5 & $\begin{array}{l}\text { Macrolides/ } \beta \text { - } \\
\text { lactams/tetracyclines/chloramphenicols/sulfonamides }\end{array}$ & $3(2.1 \%)$ & $7(5.0)$ \\
\hline \multirow[t]{4}{*}{4} & Macrolides/tetracyclines/chloramphenicols/sulfonamides & $1(0.7 \%)$ & $5(3.6 \%)$ \\
\hline & Macrolides/ $\beta$-lactams/tetracyclines/sulfonamides & $\begin{array}{l}109 \\
(77.9 \%)\end{array}$ & $52(37.1 \%)$ \\
\hline & Macrolides/ $\beta$-lactams/tetracyclines/chloramphenicols & $1(0.7 \%)$ & 0 \\
\hline & Macrolides/ß-lactams/sulfonamides/chloramphenicols & $1(0.7 \%)$ & 0 \\
\hline \multirow[t]{4}{*}{3} & Macrolides/ $\beta$-lactams/tetracyclines & $7(5.0 \%)$ & $9(6.4 \%)$ \\
\hline & Macrolides/ $\beta$-lactams/sulfonamides & $3(2.1 \%)$ & $3(2.1 \%)$ \\
\hline & Macrolides/tetracyclines/sulfonamides & $4(2.9 \%)$ & $40(28.6 \%)$ \\
\hline & Macrolides/tetracyclines/chloramphenicols & 0 & $3(2.1 \%)$ \\
\hline Total & -- & $\begin{array}{l}129 \\
(92.1 \%)\end{array}$ & $\begin{array}{l}119 \\
(85.0 \%)\end{array}$ \\
\hline
\end{tabular}

\section{MLST}

The MLSTs of the inpatient and outpatient isolates were shown in Table 4. Among the inpatient isolates, forty-five STs were detected, the predominant STs were ST271 (24.3\%, 34/140), ST320 (19.3\%, 27/140), ST81 $(7.1 \%, 10 / 140)$, ST876 (7.1\%, 10/140) and ST3397 (5.7\%, 8/140), which were mainly associated with serotypes 19F, 19A, 23F, 14 and 15B, respectively. Eighty-one STs were detected among the outpatient isolates, the predominant STs were ST81 (8.6\%, 12/140), ST271 $(7.9 \%, 11 / 140)$, and ST320 $(6.4 \%, 9 / 140)$, which were associated with serotypes $23 \mathrm{~F}, 19 \mathrm{~F}$, and $19 \mathrm{~A}$, respectively.

The population snapshot of the $S$. pneumoniae isolates as determined by eBURST analysis is shown in Fig. 1. The eBURST analysis results found five CCs and twenty-four singletons among the inpatient isolates, fifteen CCs and forty-two singletons among the outpatient isolates. CC271 was the most 
common CC bonth in the inpatient isolates and outpatient isolates. Among the inpatient isolates, thirteen STs were newly assigned $(9765,9781-9786,9790,9791$, and 10136) via MLST analysis; three of the new STs $(9765,9781$, and 9782) were novel combinations of known alleles, whereas the remainder had new local alleles (i.e., aroE 296, gdh 420, gdh 405, gdh 421, xpt 553, ddl 619, and ddl 620). Among the outpatient isolates, twenty-seven were newly assigned (10080-10106) via MLST analysis. Sixteen of the new STs (10080-10095) were novel combinations of known alleles, whereas the remainder contained new local alleles (gdh 431-433, gki 439, spi 423-425, xpt 594-595, and ddl 638-639).

The size of the dot was proportional to the number of strains included in the ST. The line represent that there was a single site mutation between the two clones. The black ST numbers represent the isolates from inpatients, the green ST numbers represent the isolates from outpatients, and the purple ST numbers represent the isolates from the two patients group.

Table 4

MLST of inpatient and outpatient $S$. pneumoniae isolates [n (\%)]

\begin{tabular}{|lll|}
\hline MLST & $\begin{array}{l}\text { Inpatient isolates } \\
(\mathbf{n}=\mathbf{1 4 0})\end{array}$ & $\begin{array}{l}\text { Outpatient isolates } \\
(\mathbf{n}=\mathbf{1 4 0})\end{array}$ \\
\hline 271 & $34(24.3 \%)$ & $11(7.9 \%)$ \\
320 & $27(19.3 \%)$ & $9(6.4 \%)$ \\
81 & $10(7.1 \%)$ & $12(8.6 \%)$ \\
876 & $10(7.1 \%)$ & $3(2.1 \%)$ \\
3397 & $8(5.7 \%)$ & $6(4.3 \%)$ \\
\hline others & $51(36.4 \%)$ & $99(70.7 \%)$ \\
\hline
\end{tabular}

\section{Discussion}

The present data showed that the serotypes 19F, 19A, 23F, and 6A were common both in the inpatient and outpatient isolates, which was similar to previous studies regarding inpatients with pneumonia and outpatients with upper respiratory infection $[5,6]$. Within the inpatient isolates, only fifteen serotypes were identified with the rate of serotypes $19 \mathrm{~A}$ and $19 \mathrm{~F}$ accounting for $53.6 \%$, and twenty-nine serotypes were identified among the outpatient isolates, with the serotypes $19 \mathrm{~A}$ and $19 \mathrm{~F}$ accounting for $21.5 \%$. The serotype distribution was more concentrated among the inpatient isolates and more comprehensive among the outpatient isolates.

The serotypes 19F and 19A were prevalent either in inpatient isolates or in outpatient isolates. These two serotypes are very common in invasive pneumococcal diseases [11-13], which could mean that these two serotypes frequently cause severe pneumococcal infections. Meanwhile, these two serotypes also showed high levels of antibiotic resistance. A previous study found that half of the penicillin-resistant 
isolates were identified as serotype $19 \mathrm{~F}$, and 47 of the serotype $19 \mathrm{~A}$ isolates were non-susceptible to cefuroxime, including $42(89.4 \%)$ that were resistant [1]. In another study of serotype $19 \mathrm{~F}$ of $S$. pneumoniae showed that the non-susceptibility rates to cefaclor and cefuroxime increased from $14.2 \%$ in 1997-1998 to more than $80 \%$ in 2010 [14]. One study from Bulgaria found that the MDR of serotype 19A was $82.7 \%$ [15]. Study from the USA reported that serotype 19A was the major MDR serotype (38.5\%) [16]. These results suggest that the prevalent of the serotypes $19 \mathrm{~F}$ and $19 \mathrm{~A}$ among the inpatient isolates and outpatient isolates may be related to the selective pressure of antibiotics.

The serotype coverage rates of PCV7, PCV10 and PCV13 were high among the inpatient isolates, meaning that the vaccine serotypes were more common among the inpatient isolates. Many research have proved that PCVs can decrease the pneumococcal carriage rate, disease morbidity and the antibiotic resistance [17-20]. If the vaccines could be universally immunized, the decreasing effect of vaccine related serotypes could occur earlier or greater in the survey on inpatient isolates. In inpatient department, the bacterial culture was a routine clinical work which ensured the strains can be obtained continuously. In the studies on evaluation of PCVs universal immunization effectiveness, the inpatient isolates would be a better scheme. More serotypes were identified in outpatient isolates, maybe, the outpatient isolates would set up more comprehensive diagram of S. pneumoniae serotype distribution, which could give more information for the evaluation of serotype replacement after the PCVs immunization.

Among the inpatient isolates, the penicillin non-susceptibility rate was $7.1 \%$ according to the nonmeningitis parenteral breakpoint (intermediate $[4 \mathrm{mg} / \mathrm{L}]$, resistant $[\geq 8 \mathrm{mg} / \mathrm{L}]$ ). The rate, however, increased to $94.3 \%$ when based on the oral penicillin breakpoint (intermediate [0.12-1 mg/L], resistant [ $\geq$ $2 \mathrm{mg} / \mathrm{L}])$, which was significantly higher than that in developed countries (22.4\%) [12]. A systematic literature review of prevalence, mechanisms, and clinical implications in S. pneumoniae resistance found that there has been a steady decline in susceptibility of $S$. pneumoniae to commonly used beta-lactams [21]. It should be noted that the lower penicillin non-susceptibility rate according to the non-meningitis parenteral breakpoint did not reflect the resistance to $\beta$-lactams, especially in tertiary hospitals where cephalosporins were used more often [22].

The non-susceptibility rates against penicillin, amoxicillin-clavulanic acid, imipenem, cefuroxime, cefaclor and trimethoprim-sulfamethoxazole as well as the MDRSP rate among the inpatient isolates were high. These findings suggested different empirical therapies under dissimilar conditions. The Chinese Medical Association recommended different treatment and antimicrobial programs for inpatients and outpatients with infectious S.pneumoniae according to the severity of their disease instead of the pathogen resistance [23], and the present study provides an epidemiological reference for this program. Actually, a seminal multicenter survey on antibiotic use in five tertiary children's hospitals showed that penicillins, macrolides, and cephalosporins were prescribed more often in the outpatient department, while second and third generation cephalosporins were more common in the inpatient department, with the fourth generation cephalosporins and carbapenems also administered. ${ }^{22}$ The present study indicated that the empirical antibiotic program should be different between the inpatients and outpatients. 
Forty-five STs were identified among the inpatient isolates, with ST271, ST320, ST81, ST876, and ST3397 as the most common STs. Eighty-one STs were detected among the outpatient isolates, with ST81, ST271, and ST320 were the most common STs. The MLSTs in two groups of isolates were consistent with those of a previous study [24]. Twenty-five STs were only identified among the inpatient isolates. As we all known, the inpatients' illness condition was more seriously, it may meant that the STs were related to the disease severity. One study from India found that the STs of the invasive and nasopharyngeal carriage isolates were different. ST 63, ST4219, ST236, ST11921 and ST3135 were more common among invasive isolates, while ST4894, ST1701 and ST236 were more common among nasopharyngeal carriage isolates [25]. There was a survey of epidemiological data showing trends toward the association of the ancestral type of the capsular regulatory genome with carriage and the association of laterally transferred sequences with invasive disease isolates [26]. All of the aforementioned results indicated that the microbiology characteristics were related to the pathogenicity of $S$. pneumoniae.

Similar to the serotype distribution, the STs were more concentrated among the inpatient isolates. ST271 and ST320 were high among the inpatient isolates which were associated with serotypes 19F and 19A, respectively. CC271 was the most common CC in the two groups which include ST271, ST320, ST236, and ST4314. According to the data of Pneumococcal Molecular Epidemiology Network (https://pubmlst.org/spneumoniae), ST320 belonged to Taiwan ${ }^{19 F_{-}} 14$, and the prevalence of resistant CC might be the main reason of high levels of antimicrobial resistance among the inpatient isolates. We really need to take more effective measures to release the antibiotics resistance of $S$. pneumoniae.

The present study had several limitations. This was a single-center study, and the clinical background information such as gender, diagnosis and treatment were unavailable. We just describe the epidemiological characteristics of $S$. pneumoniae isolated from inpatients and outpatients. Longitudinal and multicenter surveillance with more background information of pneumococcal isolates is necessary to confirm the present results and evaluate the influence of PCVs universal immunization in the future.

\section{Abbreviations}

PCR: Polymerase chain reaction; MLST: Multilocus sequence typing; CC: Clonal complex; MDR: Multi-drug resistant; MIC: Minimum inhibitory concentration.

\section{Declarations}

\section{Competing interests}

The authors declare that they have no competing interests.

\section{Authors'Contributions}

All of the authors had full access to the full dataset (including the statistical reports and tables) and take responsibility for the integrity of the data and the accuracy of the analysis. YKH, YYH, LS, XBP, DF, LG, and 
WQ conceived and designed the study. LS, XBP, DF, LG, WQ, and SW collected the data and developed the analysis. YKH and LS interpreted the data. YKH and LS wrote the first draft of the paper. YKH and YYH reviewed and approved of the final report.

\section{Acknowledgements}

Not Applicable.

\section{Founding}

The National Natural Science Foundation of China (Grant No. 81371853), the Capital Health Research special development (Grant No. 2014-2-1141) and the National Clinical Center Project of the Ministry of Science and Technology of the People's Republic of China (Grant No. 2013BAI09B11) financially supported this study. The funding sources had no role in the study design, data collection and analysis, decision to publish, or preparation of the manuscript.

\section{Ethics approval and consent to participate}

A parent or legal guardian of each participant signed a written informed consent document before enrollment and before any study procedure was performed. This study was reviewed and approved by the Ethics Committee at Beijing Children's Hospital Affiliated to Capital Medical University. Ethical problems were not encountered in this study.

\section{References}

1. Xue L, Yao K, Xie G, Zheng Y, Wang C, Shang Y, et al. Serotype distribution and antimicrobial resistance of Streptococcus pneumoniae isolates that cause invasive disease among Chinese children. Clin Infect Dis. 2010;50(1):741-4.

2. Centers for Disease Control and Prevention. Defining the public health impact of drug-resistance Streptococcus pneumoniae: report of a working group. MMWR Recomm Rep. 1996;45(RR-1):1-20.

3. Satzke C, Turner P, Virolainen-Julkunen A, Adrian PV, Antonio M, Hare KM, et al WHO Pneumococcal Carriage Working Group. Standard method for detecting upper respiratory carriage of Streptococcus pneumoniae: updated recommendations from the World Health Organization Pneumococcal Carriage Working Group. Vaccine. 2013;32(1):165-79.

4. Yao KH, Wang LB, Zhao GM, Zheng YJ, Deng L, Huang JF, et al. Pneumococcal serotype distribution and antimicrobial resistance in Chinese children hospitalized for pneumonia. Vaccine. 2011;29(12):2296-301.

5. Yao K, Shen X, Yul S, Lu Q, Deng L, Ye Q, et al. Antimicrobial resistance and serotypes of nasopharyngeal strains of Streptococcus pneumoniae in Chinese children with acute respiratory infections. J Int Med Res. 2007;35(2):253-67. 
6. Zhou L, Yu SJ, Gao W, Yao KH, Shen AD, Yang YH. Serotype distribution and antibiotic resistance of 140 pneumococcal isolates from pediatric patients with upper respiratory infections in Beijing, 2010. Vaccine. 2011;9:7704-10.

7. Sorensen UB. Typing of pneumococci by using 12 pooled antisera. J Clin Microbiol. 1993;31(8):2097-100.

8. Wayne P. Performance standards for antimicrobial susceptibility testing: Twenty Fifth International Supplement M100-S25. Clinical and Laboratory Standards Institute; 2015.

9. Enright MC, Spratt BG. A multi locus sequence typing scheme for Streptococcus pneumoniae: identification of clones associated with serious invasive disease. Microbiology. 1998;144(Pt11):3049-60.

10. McGee L, McDougal L, Zhou J, Spratt BG, Tenover FC, George R, et al. Nomenclature of major antimicrobial-resistant clones of Streptococcus pneumoniae defined by the pneumococcal molecular epidemiology network. J Clin Microbiol. 2001;39:2565-71.

11. Lo SW, Gladstone RA. van Tonder AJ, Lee JA, du Plessis M, Benisty R. et al. Global Pneumococcal Sequencing Consortium. Pneumococcal lineages associated with serotype replacement and antibiotic resistance in childhood invasive pneumococcal disease in the post-PCV13 era: an international whole-genome sequencing study. Lancet Infect Dis 2019;19(7):759 - 69.

12. Beall B, Chochua S, Gertz RE Jr, Li Y, Li Z, McGee L. er al. A Population-Based Descriptive Atlas of Invasive Pnemococcal Strains Recovered Within the U.S. During 2015-2016. Front Microbiol. 2018;19(9):2670.

13. Hackel M, Lascols C, Bouchillon S, Hilton B, Morgenstern D, Purdy J. Serotype prevalence and antibiotic resistance in Streptococcus pneumoniae clinical isolates among global populations. Vaccine. 2013;31:4881-7.

14. Li QH, Yao KH, Yu SJ, Ma X, He MM, Shi W, et al. Spread of multidrug-resistant clonal complex 271 of serotype 19F Streptococcus pneumoniae in Beijing, China: characterization of serotype 19F. Epidemiol Infect. 2013;141:2492-6.

15. Setchanova LP, Alexandrova A, Dacheva D, Mitov I, Kaneva R, Mitev V.. Dominance of MultidrugResistant Denmark 14-32 (ST230) Clone Among Streptococcus pneumoniae Serotype 19A Isolates Causing Pneumococcal Disease in Bulgaria from 1992 to 2013. Microb Drug Resist. 2015;21(1):3542.

16. Richter SS, Diekema DJ, Heilmann KP, Dohrn CL, Riahi F, Doern GV. Changes in Pneumococcal Serotypes and Antimicrobial Resistance After Introduction of the 13-Valent Conjugate Vaccine in the United States. Antimicrob Agents Chemother. 2014;58(11):6484-9.

17. Løvlie A, Vestrheim DF, Aaberge IS, Steen A. Change in pneumococcal carriage prevalence and factors associated with carriage in Norwegian children, four years after introduction of PCV13. BMC Infect Dis. 2020;20(1):29.

18. Dondo V, Mujuru H, Nathoo K, Jacha V, Tapfumanei O, Chirisa P. Pneumococcal Conjugate Vaccine Impact of Meningitis and Pneumonia Among Children Agedī1\%४5 Years-Zimbabwe, 2010-2016. Clin 
infect Dis. 2019;69(2):72-80.

19. Nakano S, Fujisawa T, Ito $Y$, Chang B, Matsumura $Y$, Yamamoto M. Nationwide surveillance of paediatric invasive and non-invasive pneumococcal disease in Japan after the introduction of the 13-valent conjugated vaccine, 2015-2017. Vaccine. 2019;38(7):1818-24.

20. Schroeder MR, Chancey ST, Thomas S, Kuo WH, Satola SW, Farley MM, Stephens DS. A Population Based Assesment of the Impact of 7- and 13-Valent Pneumococcal Conjugate Vaccine on Macrolide-Rsistant Invasive Pneumococcal Disese: Emergence and Decline of Streptococcus pneumoniae Serotype 19A (CC320) With Dual Macrolide Resistance Mechanisms. Clin Infet Dis. 2017;65(6):990-8.

21. Cherazard R, Epstein M, Doan TL, Salim T, Bharti S, Smith MA.. Antimicrobial Resistant Sreptococcus pneumoniae: Prevalence, Mechanisms, and Clinical Implications. Am J Ther. 2017;24(3):e361-9.

22. Zhang $W$, Shen $X$, Wang $Y$, Chen $Y$, Huang $M$, Zeng $Q$, et al. Antibiotic consumption in five tertiary children's hospitals before and after implementation of guidelines for antibacterial use in clinical practice. Chin J E id Based Pediatr. 2010;15(6):404-11. (in Chinese).

23. Chinese Medical Association Branch of Pediatrics, China Preventive Mediceine Association. Guidelines for the prevention and treatment of pediatric Streptococcus pneumonia infectious diseases. Chin J Pediatr. 2010;48(2):104-11. (in Chinese).

24. Qian J, Yao KH, Xue L, Xie G, Zheng Y, Wang C, et al. Diversity of pneumococcal surface protein A (PspA) and relation to sequence typing in Streptococcus pneumoniae causing invasive disease in Chinese children. Eur J Clin Microbiol Infect Dis. 2011;31(3):217-23.

25. Varghese R, Neeravi A, Subramanian N, Pavithra B, Kavipriya A, Kumar JL, et al. Clonal similarities and sequence-type diversity of invasive and carriage Streptococcus pneumoniae in India among children under 5 Years. Indian J Med Microbiol. 2019;37(3):358 - 62.

26. Varvio SL, Auranen K, Arjas E, Mäkelä PH. Evolution of the Capsular Regulatory Genes in Streptococcus pneumoniae. J Infect Dis. 2009;200:1144-51.

\section{Figures}




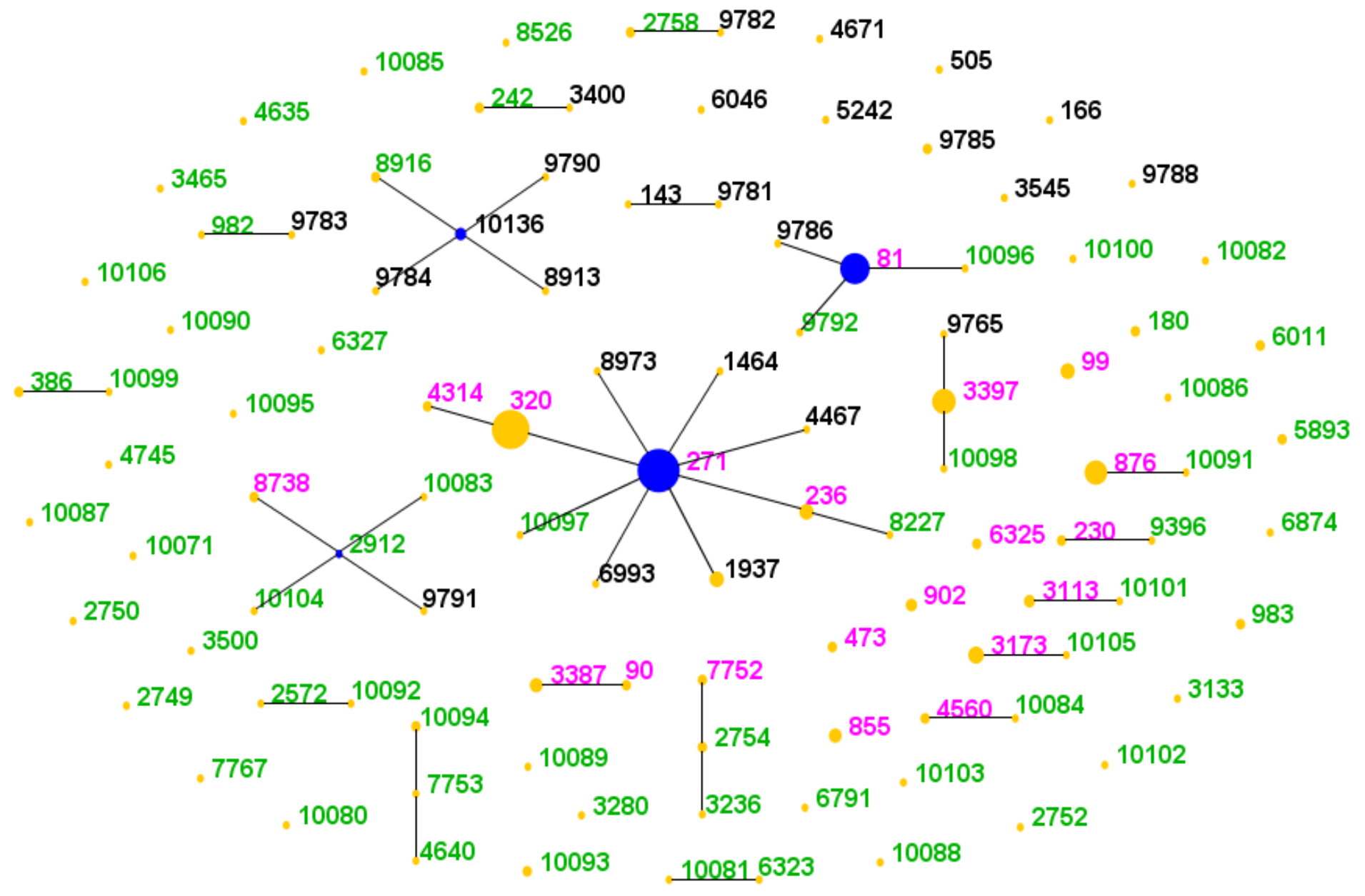

Figure 1

A population snapshot of the inpatient and outpatient S. pneumoniae isolates via eBURST analysis. 